\title{
CASMIL: a comprehensive software/toolkit for image-guided neurosurgeries $^{\dagger}$
}

\author{
Gulsheen Kaur, ${ }^{1}{ }^{*}$ Jun Tan, ${ }^{2}$ \\ Mohammed Alam, ${ }^{1}$ \\ Vipin Chaudhary, ${ }^{1,6}$ \\ Dingguo Chen, ${ }^{2}$ Ming Dong, ${ }^{1}$ \\ Hazem Eltahawy, ${ }^{3}$ \\ Farshad Fotouhi, ${ }^{1}$ \\ Christopher Gammage, ${ }^{1}$ \\ Jason Gong, ${ }^{3}$ William Grosky, ${ }^{5}$ \\ Murali Guthikonda, ${ }^{1,3}$ \\ Jingwen $\mathrm{Hu}^{1}$ \\ Devkanak Jeyaraj, ${ }^{1}$ Xin Jin, ${ }^{1}$ \\ Albert King, ${ }^{1}$ Joseph Landman, ${ }^{7}$ \\ Jong Lee, ${ }^{1}$ Qing Hang Li, ${ }^{1,3}$ \\ Hanping Lufei, ${ }^{1}$ Michael Morse, ${ }^{4}$ \\ Jignesh Patel, ${ }^{4}$ Ishwar Sethi, ${ }^{2}$ \\ Weisong Shi, ${ }^{1}$ King Yang, ${ }^{1}$ \\ Zhiming Zhang, ${ }^{1}$ \\ ${ }^{1}$ Computer-assisted Surgery \\ Laboratory, Wayne State University, \\ Detroit, MI, USA \\ ${ }^{2}$ Oakland University, Rochester Hills, \\ MI, USA \\ ${ }^{3}$ The Detroit Medical Centre, Detroit, \\ MI, USA \\ ${ }^{4}$ University of Michigan, Ann Arbor, \\ MI, USA \\ ${ }^{5}$ University of Michigan, Dearborn, \\ MI, USA \\ ${ }^{6}$ Institute for Scientific Computing, \\ Detroit, MI, USA \\ ${ }^{7}$ Scalable Informatics LLC, Canton, \\ MI, USA
}

*Correspondence to: Gulsheen Kaur, Computer-assisted Surgery

Laboratory, Wayne State University, Detroit, MI, USA. E-mail:

gkaur@med.wayne.edu

${ }^{\dagger}$ This research was supported in part by a research grant from Michigan Life Sciences Corridor (Grant No MEDC-459).

Accepted: 30 March 2006

\begin{abstract}
Background CASMIL aims to develop a cost-effective and efficient approach to monitor and predict deformation during surgery, allowing accurate, and real-time intra-operative information to be provided reliably to the surgeon.
\end{abstract}

Method CASMIL is a comprehensive Image-guided Neurosurgery System with extensive novel features. It is an integration of various modules including rigid and non-rigid body co-registration (image-image, image-atlas, and image-patient), automated 3D segmentation, brain shift predictor, knowledge based query tools, intelligent planning, and augmented reality. One of the vital and unique modules is the Intelligent Planning module, which displays the best surgical corridor on the computer screen based on tumor location, captured surgeon knowledge, and predicted brain shift using patient specific Finite Element Model. Also, it has multi-level parallel computing to provide near real-time interaction with iMRI (Intra-operative MRI). In addition, it has been securely web-enabled and optimized for remote web and PDA access.

Results A version of this system is being used and tested using real patient data and is expected to be in use in the operating room at the Detroit Medical Center in the first half of 2006.

Conclusion CASMIL is currently under development and is targeted for minimally invasive surgeries. With minimal changes to the design, it can be easily extended and made available for other surgical procedures. Copyright (c) 2006 John Wiley \& Sons, Ltd.

Keywords intelligent planning; computer-assisted surgery; neurosurgery; finite element model; parallel computing in surgery; augmented reality; brain shift

\section{Introduction}

Computer-assisted surgery (CAS) is a methodology that translates into accurate and reliable image-to-surgical space guidance. Neurosurgery is a very complex procedure and the surgeon has to integrate multi-modal data to produce an optimal surgical plan. Often the lesion of interest is surrounded by vital structures, such as the motor cortex, temporal cortex, vision and audio sensors, etc., and has irregular configurations. Slight damage to such eloquent brain structures can severely impair the patient $(1,2)$. CASMIL, an imageguided neurosurgery toolkit, is being developed to produce optimum plans resulting in minimally invasive surgeries. This system has many innovative features needed by neurosurgeons that are not available in other academic and commercial systems. CASMIL is an integration of various vital modules, such as rigid and non-rigid co-registration (image-image, image-atlas and 


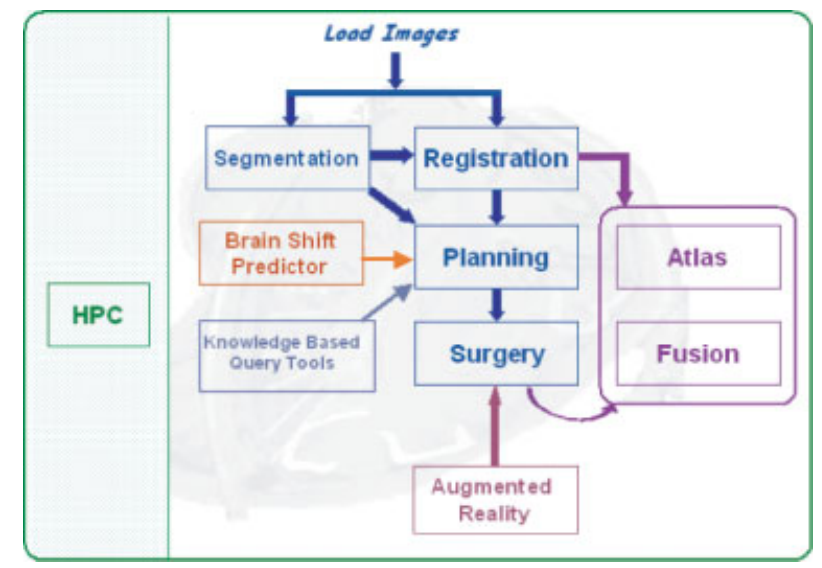

Figure 1. CASMIL modules and flow

image-patient), 3D segmentation, brain shift predictor (BSP), knowledge-based query tools, intelligent planning and augmented reality.

Figure 1 shows the flow of steps involved in computerassisted surgery. For any neurosurgical procedure, the first step is always image acquisition, followed by loading of acquired sequences in an image-guided system (CASMIL in our case). Next, we can either perform segmentation of relevant structures or co-registration among different image modalities. Once the structure of interest, e.g. a tumour, has been segmented, planning can be performed. The intelligent planning module of CASMIL utilizes the brain shift predictor and knowledge-based query tools modules as input. The digital image atlas is also loaded and co-registered with the anatomical image sequence. The intelligent planning module can be used both preoperatively and intraoperatively. The augmented reality module can be used intraoperatively to obtain a composite view of 3D real environment and graphical images. For all the modules, high-performance computing (HPC) is utilized for computationally intensive steps to speed up the process. All these modules are described in detail in the section on CASMIL modules, below.

As part of this project, Detroit Medical Centre has acquired a portable intraoperative magnetic resonance imaging system (iMRI; with $0.15 \mathrm{~T}$ from Odin Medical Technologies) that is currently being used. We have access to the iMRI data for research purposes and we are developing a database (CASMILDB) that is being populated with the performed iMRI cases. These cases are used for validation and continuous improvement of the brain shift predictor module. CASMILDB stores multimodal information, such as different image modalities, surgical approaches, best slices, segmented objects, etc. Currently, CASMILDB is being populated with 3000 cases from the hospital repository.

This paper is organized as follows. The next section briefly describes the component design of CASMIL. The following section details the various modules in CASMIL. A review of the related work and comparison with CASMIL are then given.

\section{CASMIL design}

CASMIL has been developed in a structured manner. Components with different functions are developed as blocks that can be simply inserted in the main framework to work together. We have defined the interface between the main framework and its components. By following the interface, we can integrate additional functions with CASMIL easily. For example, each segmentation algorithm is implemented as a class inherited from a base class, which is called 'XSegImage'. To add a new segmentation algorithm, such as 'Simple fuzzy connectedness', we can simply inherit a new class 'XSimpleFuzzy' from 'XSegImage', add parameters 'm_SamplePoints' and ' $m \_S e e d P o i n t s '$ ', and instantiate its virtual function 'Segment()'. The segmented output will be implicitly converted to CASMIL internal format by the base class 'XSegImage' and the main GUI process will display it. Figure 2 shows a subset of the relationship between the base class and the inheritances in CASMIL.

The current version of CASMIL has been optimized for neurosurgery. However, with minimal changes it can have broad applicability to a wide variety of fields, including other surgical specialties, radiation oncology, and biological and medical research. In addition, the developed modules are scalable and can be used in many other biomedical applications, such as computer-assisted diagnostic imaging and database mining. This system has been developed using VC++, Qt and OpenGL on a Windows platform and has been designed to work independently of the operating system. As we describe later, it executes on a Linux-based palm device.

\section{CASMIL modules}

This section provides a brief overview of the modules integrated in CASMIL. Before activating any module, all image sequences related to a case need to be loaded. CASMIL can load standard DICOM (MRI, CT, PET, etc.) or IMG image sequences and can display multi-planar, threedimensional (3D) and video views (Figure 3). Other tools, such as contrast and brightness adjustment, panning, rotation by angle and flipping, are also included. Images can either be rendered as greyscale images or as coloured images. For displaying coloured images, 16 different colour maps have been implemented.

\section{Co-registration}

Image registration is the process of aligning images for one-to-one correspondence among various features. In the medical imaging domain, this could involve aligning images from the same modality, MR-MR, multi-modality MR-CT, MR-PET, MR-DTI, etc. Image registration can be classified broadly into rigid, non-rigid, and model-based registrations. 


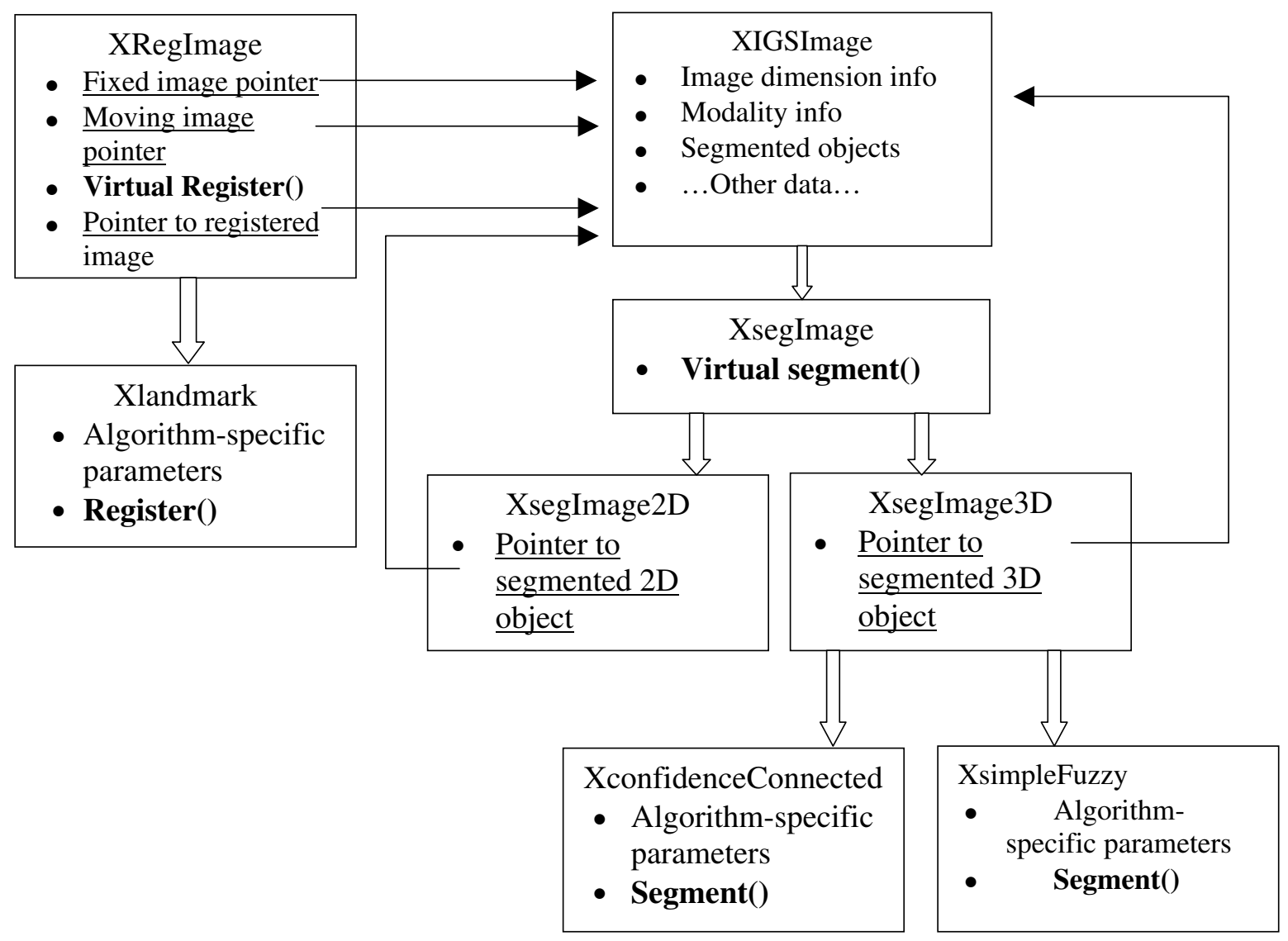

Figure 2. Class inheritance in CASMIL

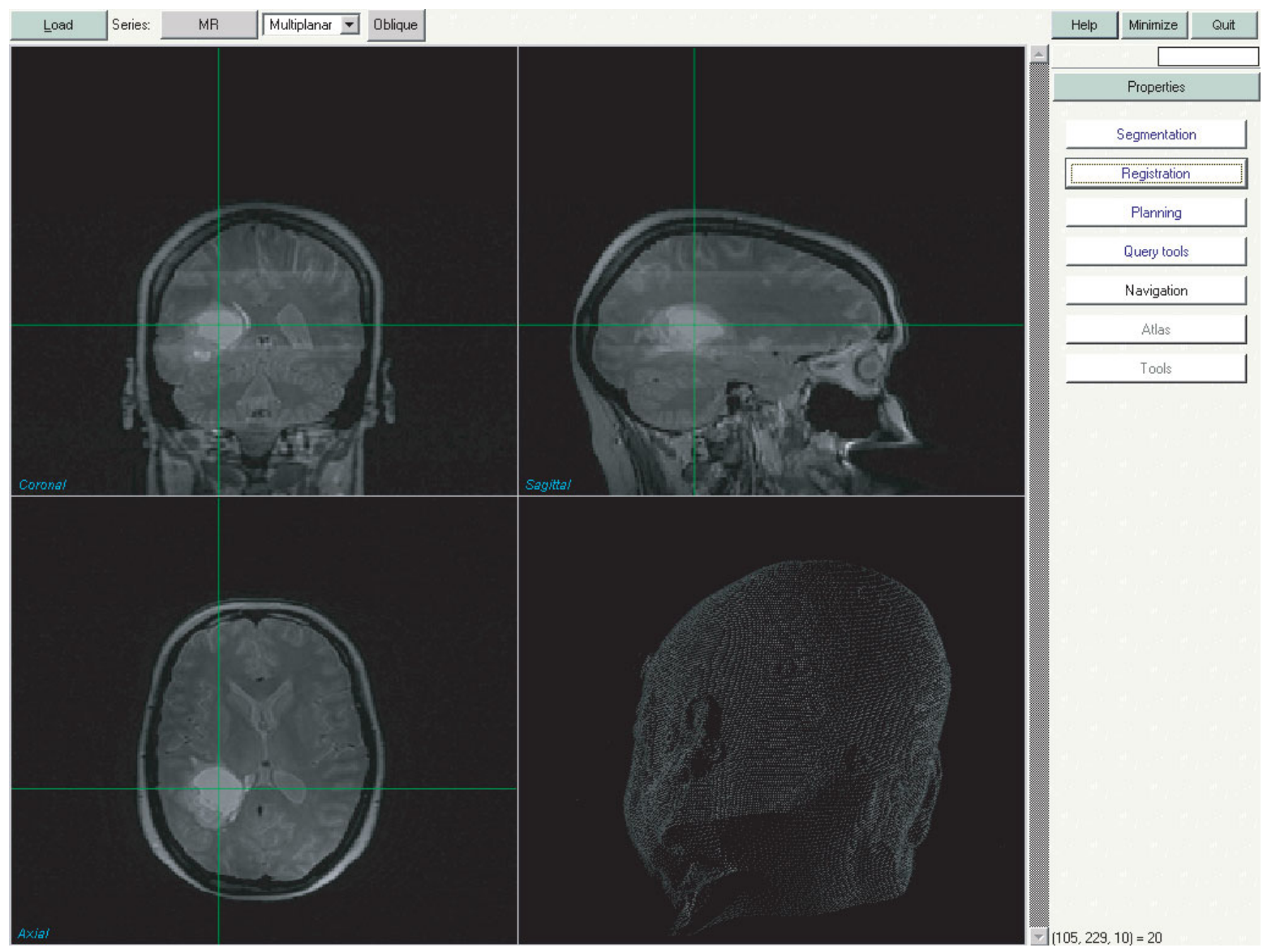

Figure 3. Multi-planar view 
In the rigid framework, the misalignment can usually be characterized by translation and rotation parameters. The rigid framework assumes that there are no considerable changes with the brain adopting different poses. In the current CASMIL version, five registration algorithms, viz. landmark-based, multi-resolution mutual information, hybrid of landmark and mutual information, landmark warping and viola wells have been integrated (3). These techniques have been selected on the basis of ease of use, surgeon recommendation, human interaction, computational time required, and accuracy.

In addition, CASMIL includes more complex registration (NRR) algorithms: Thirion's 'demons' algorithm (3-5) and geometrical model-based registration (3). Other non-rigid algorithms that are being integrated with CASMIL are: diffeomorphic landmark matching (6), diffeomorphic flow-based image registration (7), and deformation registration from the Lagrangian frame (8). Non-rigid registration is now a standard component for advanced image registration algorithms, which range from fast, basic image-processing algorithms to highly advanced physical models that push the boundaries of numerical simulation and computation. Compared to rigid registration, non-rigid registration is more efficient, as it directly incorporates optimization into implementation and is sensitive to much localized differences, yet exhibits specificity and robustness. The classical philosophy of non-rigid image registration is to model an image as a continuum (fluid, plastic, elastic, etc.) The medium is then allowed to deform in order to satisfy some a priori optimization criterion.

In Thirion's 'demons' algorithm, each image is viewed as a set of iso-intensity contours. The main idea is for small 'demons' to 'push' the image around by its level sets until correspondence is achieved. The orientation and magnitude of the displacement is derived from the instantaneous optical flow equation:

$$
D(x) \cdot \nabla f(x)=-(m(x)-f(x))
$$

Here $m(x)$ is moving image, $f(x)$ is fixed image and $D(x)$ is the displacement or optical flow between the images. $D(x)$ could be normalized as:

$$
D(x)=\frac{-(m(x)-f(x)) \nabla f(x)}{\|\nabla f\|^{2}+(m(x)-f(x))^{2}}
$$

Starting from the initial deformation field, the displacement vector will be updated for each iteration using the above equation until a certain criterion is met.

In geometrical model-based registration, a geometrical model is warped into an image based on morphological information by identifying a number of parameters in the model. Model parameters are optimized until the model comes into a good representation of the anatomical structures contained in an image. The core components of this registration framework are: the basic input data (pixel data from an image) and geometrical data from a spatial object. A metric has to be defined in order to evaluate the fit between the model and the image. This transformation is the variations in the spatial positioning of the spatial object and/or by changing its model parameters. The search space for the optimizer is now the composition of the transformed parameter and the shape of the internal parameters (3).

\section{Image-atlas co-registration}

CASMIL can also perform image-atlas registration. It uses Schaltenbrand (SW), Talairach and Tournoux (TT88) and Talairach and Tournoux (TT93) digitized brain atlases (9-12). The transfer of anatomical knowledge from 3D atlases to patient images via image-atlas co-registration is a very helpful tool for planning. However, there are anatomical differences among individual patients that make registration difficult. An accurate voxel-wise fusion of different individuals is hardly possible. For planning and simulation applications, accuracy is essential, because any geometrical deviation may be detrimental to a patient. Landmark-based registration is one of the most popular algorithms in atlas-based application $(12,13)$. CASMIL integrates landmark-based registration as its first atlas registration algorithm. Here, Anterior Commissure, Posterior Commissure, Left, Right (AC, PC, L, R) are chosen as control points (14). Linear conformal is then used to perform global spatial transformation. MRI is our fixed image and atlas TT 88 axial view is our moving image. The registration result is demonstrated in Figure 4.

CASMIL also integrates a volume-rendered brain atlas (15). The 3D brain atlas visualization and construction provides efficient tools to process and analyse 3D images, object boundaries, 3D models and other associated data items in an easy-to-use environment. It is the standard template of 3D brain structures, which allows us to define brain spatial characteristics, such as position of a structure, feature relevance, shape characteristics, etc. Our research is based on the following technical objectives:

1. Focus on the structural and functional organization of the brain. In humans and other species, the brain's complexity and variability across subjects is so enormous that reliance on atlases is critical to manipulate, analyse and interpret brain data effectively.

2. Describe one or more aspects of brain structures and their relationships after applying appropriate registration and warping strategies, indexing schemes and nomenclature systems. Atlases made from multiple modalities and individuals provide the capability to describe image data with statistical and visual power.

3. Provide user-friendly segmentation and labelling of patient-specific data.

Cerefy atlas consists of three orthogonal stacks of twodimensional cross-sections through a brain, which are 

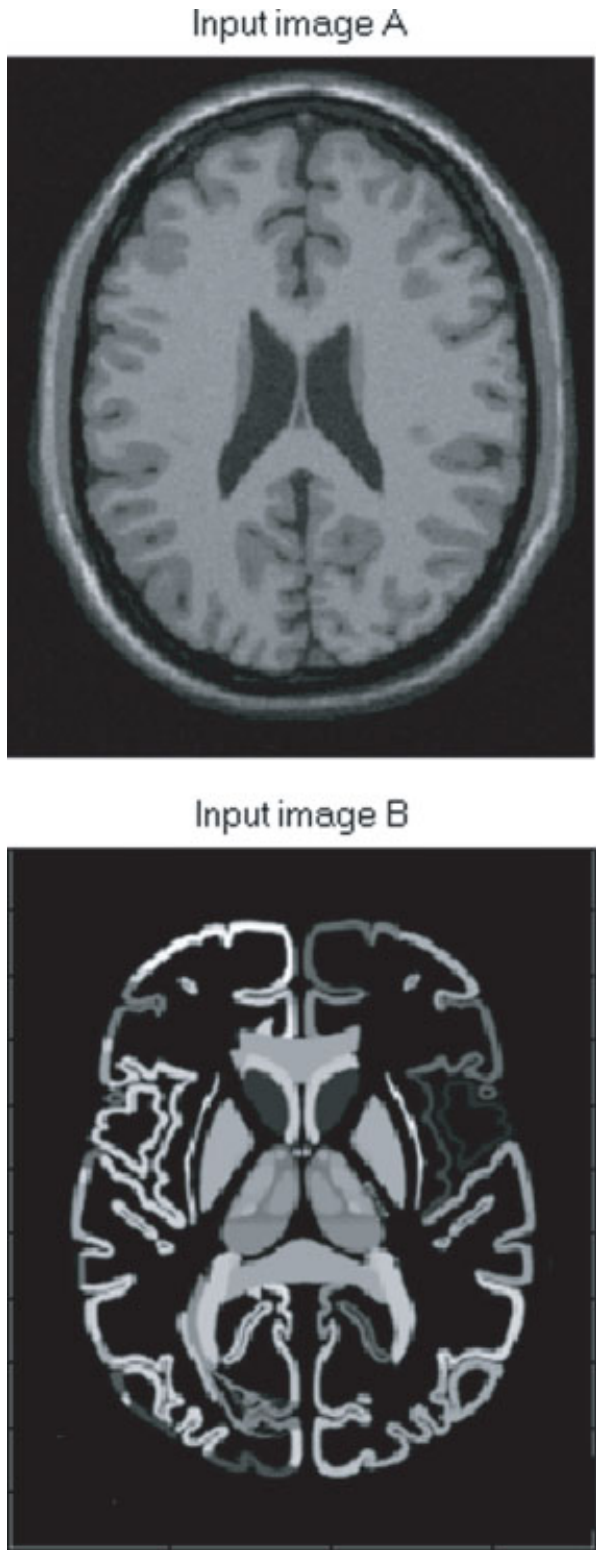

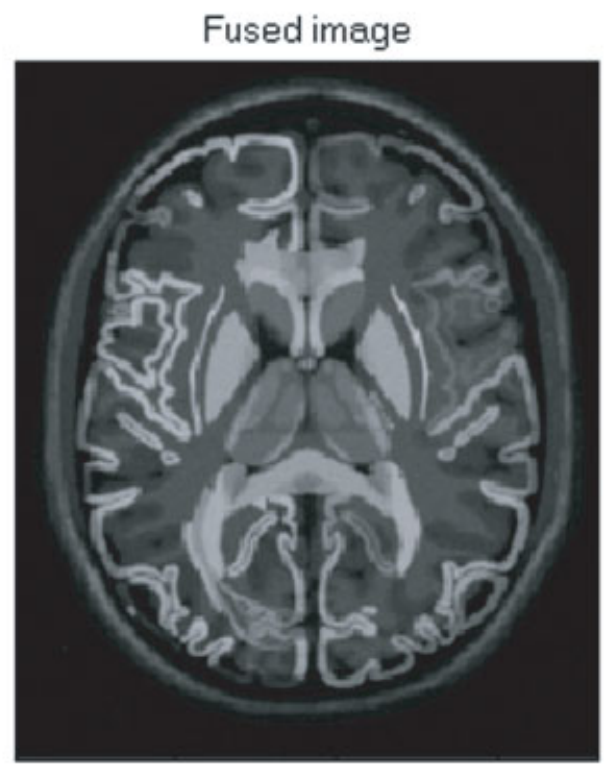

Save fused image Fuse Now !

Fusion method

Average

Figure 4. Snapshot of landmark-based patient and digital atlas co-registration

orientated sagittally, coronally and axially. The inter-slice distance is $2-5 \mathrm{~mm}$. We calculated a $3 \mathrm{D}$ reconstruction of all objects contained in the atlas at their appropriate location. The proposed method interpolates additional cross-sections between each pair of adjacent original plates. To interpolate the shape of an object for the new cross-section, we calculate a Delaunay tetrahedrization of the object using the Nuages algorithm proposed by Geiger (16). The shell surface of the resulting solid is then intersected at half the slice distance. The original stacked and interpolated slices can be considered as a binary voxel space, where the grey value of each voxel indicates the structure to which the voxel belongs. The algorithm structure for Delaunay reconstruction is as follows:

1. BEGIN DELAUNAY RECONSTRUCTION

2. INPUT: A 3D polygonal reconstruction

3. OUTPUT: A 3D polygonal reconstruction a. For $\mathrm{i}=0$ to number of cross-sections

i. $\mathrm{Si}=$ set of vertices in plane

ii. $\mathrm{Ci}=$ the contour edges in plane

iii. Dti = 2D_DELAUNAY (Si)

iv. ADD-VERTICES (DTi,Ci)

b. For $\mathrm{i}=0$ to number of cross-sections -1

i. $\mathrm{G}=3 \mathrm{D}$-DELAUNAY(DTi, Dti +1 )

ii. REMOVE_TETRAS (G)

iii. OUTPUT (tetrahedra, surface triangles)

\section{END DELAUNAY RECONSTRUCTION}

Smoothing of the terraced shapes has been achieved by applying a spatial smoothing filter in plane, and calculating the mean of two adjacent slices. We then deduce the enormous number of surface triangles produced by this method by applying the polygon reduction algorithm proposed by Melax (17). By this means the number of vertices will be reduced to $50 \%$ without noticeable loss of quality. The edge cos $t$ function 
is as follows:

$$
\begin{aligned}
& \cos t(u, v)=\|u-v\| \\
& \times \max _{f \in T_{u}}\left\{\min _{n \in T_{u v}}\{(1-f \cdot \text { normal } \bullet n \cdot \text { normal }) \div 2\}\right\}
\end{aligned}
$$

By this method, we can balance the balance curvature and size when determining the edge to collapse; and it works well on ridges.

CASMIL includes 3D co-registration of the volumerendered brain atlas with volume-rendered anatomical image modalities (MRI, CT, fMRI, DTI, PET, etc.).

Repeated research articles and vast clinical practice have shown that the proportional grid transformation we are using presents satisfying registration results. However, it still has some limitations: it does not handle boundary areas and highly variable cortical areas well. It also may not work well if the brain has large deformations due to brain lesions. To overcome these limitations, we have proposed a model-based registration in the next step of our research, which will provide more accurate mapping outcome in these areas by evolving edges. We will also provide a graphical user interface (GUI) in our system, which will allow users to manually improve the registration result by expert knowledge.

The error assessment and validation process of imageatlas co-registration will be performed in three different ways:

1. Expert knowledge will be used to study and match accuracy between the cross-sections of constructed 3D model and original atlas slices.

2. Two functional MRI examinations (the precentral gyrus and putamen) will be performed to assess quantitative measures of reconstruction and matching accuracy.

3. Based on a sequence of different experiments, statistical analysis will be applied to determine the low and high error boundaries.

\section{Segmentation}

Before surgery, surgeons first define volumes of interests (VOI's), such as lesions, ventricles, etc., which is referred to as segmentation. Segmentation is the term used to refer to the partitioning of an image into relatively homogeneous regions. From this partitioned image the object or the regions of interest can be separated from the background. In CASMIL, we have implemented five segmentation algorithms: Connected Threshold, Confidence Connected, Neighbourhood Connected, Simple Fuzzy Connected and 3D Confidence Connected (3). Figure 5 shows a snapshot of 3D segmentation of a tumour and the ventricles. A set of manual tools has been provided to refine the segmented object, if needed. Various segmentation techniques have been integrated because no single approach can generally solve the problem of segmentation for a large variety of image modalities.

In addition, CASMIL integrates automated segmentation. The automated anatomical labelling and comparative morphometric analysis of brain imaging is being performed by warping a prelabelled atlas into congruence with the subject anatomy (15). The strategy emphasizes anatomically meaningful atlas deformations in the presence of strong degeneration and substantial morphological differences. The atlas deformation is not driven by image intensity similarities but by continuous anatomical correspondence maps, derived from individual presegmented brain structures of standard digital brain atlases. This approach has the potential to eliminate the difficulties of manual segmentation, and substructure segmentation is positively time saving for an adequate patient sample size. It is relatively easier to obtain consistent segmentation between individual patients and for the same patient over time.

Another automated segmentation integrated with CASMIL is the ratio-based automatic multilevel thresholding selection method. The method is based on an intuition that there are some natural threshold choices for an image and that these can be located by studying the behaviour of the ratio of the number of pixels below the threshold to the number of pixels above the threshold. We demonstrated the accuracy of our method by using the selected thresholding values in a connected thresholding filter with post-processing, using morphological operation to perform segmentation of multi-modal brain tissue sequences. Also, we compared the threshold values

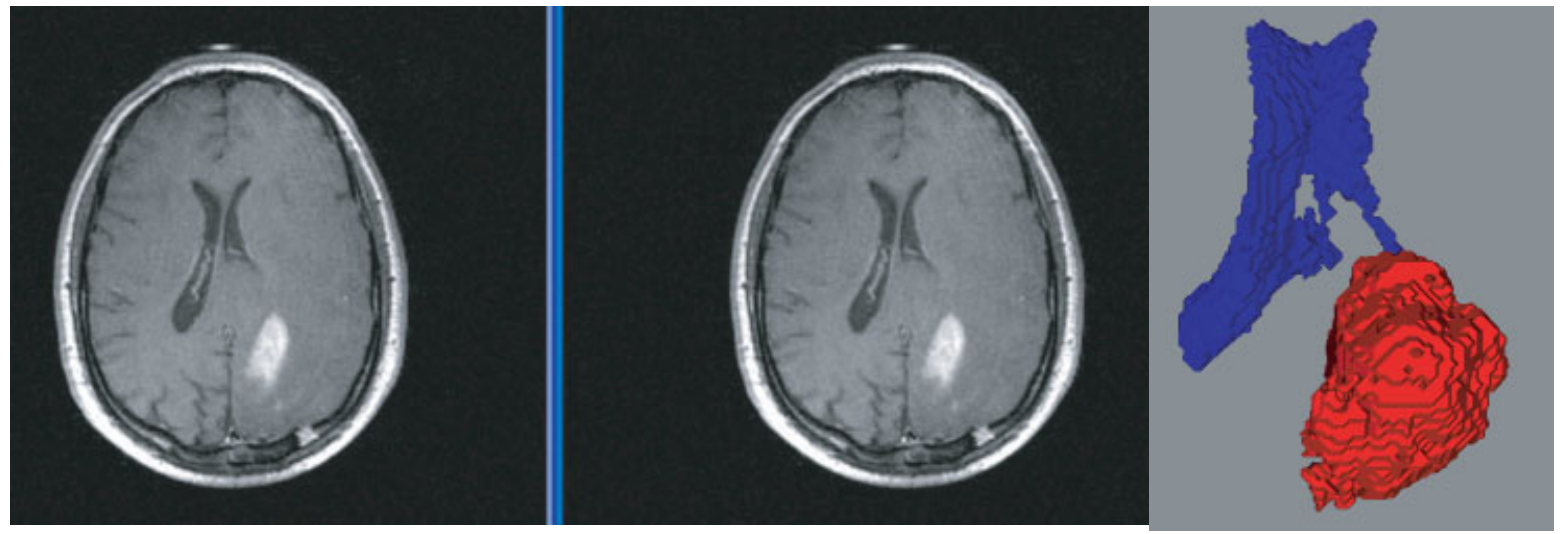

Figure 5. Snapshot of anatomical MRI DICOM slices and 3D segmented ventricles and tumour 
provided by our algorithm against the optimal threshold values under the Gaussian distribution for grey level values. A quantitative cross-validation on Brainweb discrete phantom has been provided, using three performance criteria to demonstrate the efficacy of our approach.

\section{Knowledge-based query tools}

CASMIL utilizes multi-modal data during a neurosurgical procedure. We have developed a database, CASMILDB, using MS SQL for storing multi-modal information such as different image modalities, surgical approaches, best slices, segmented objects, etc. An overview of the database is shown in Figure 6. The schema is flexible, enabling multiple modalities of data to be easily amended for future patients. Currently CASMILDB is being populated with 3000 cases from the hospital repository. In addition to imaging data, this database is also populated with surgeon knowledge in terms of the weights assigned to brain structures. Weights have been assigned and validated by neurosurgeons on a scale of $1-5,5$ being critical, as indicated in Figure 7 in red. Utilizing the CASMILDB, CASMIL can perform extensive queries to assist the surgeon both preoperatively and intraoperatively. This results in automatic real-time identification of structures and automated atlas integration. Examples of the queries include:

- Retrieve planning (entry and target coordinates) of all cases with frontally located tumours (parietal, occipital, temporal, etc.).

- Retrieve all previous cases with type of tumour (low-grade glioma, anaplastic, astrocytoma, glioblastoma, multiforme, meningioma, neurinoma, metastatic tumour, etc.).

- Retrieve all cases with frontally located tumour (parietal, occipital, temporal, etc.).
- Retrieve all previous cases with type of tumour (low-grade glioma, anaplastic, astrocytoma, glioblastoma, multiforme, meningioma, neurinoma, metastatic tumour, etc.) and located at different brain regions (frontal, parietal, occipital, temporal, etc.).

- Retrieve brain structure (ventricles, corpus callosum, motor cortex, white matter, grey matter, etc.) from brain atlas.

- Retrieve all cases that have MRI-T1 (T2, CT, PET, DTI etc.) and MRI-T2 (T1, CT, PET, DTI etc.).

- Retrieve all cases that have MRI-T1 (T2, CT, PET, DTI etc.) and MRI-T2 (T1, CT, PET, DTI etc.) and segmented brain structures (ventricles, corpus callosum, motor cortex, white matter, grey matter, etc.).

- Retrieve best slices (slices with highlighted tumour) for a particular case.

\section{Brain shift predictor}

Most of the current neuro-navigational systems are only based on preoperative images; as a result, intraoperative brain shift significantly affects the efficacy of these neuronavigational systems. CASMIL compensates brain shift by using a patient-specific finite element (FE) model being developed by our group $(18,19)$, which will preoperatively predict the direction and magnitude of brain shift, based on tumour location and surgical corridor. This model is developed with detailed anatomical structures (grey and white matter, ventricles, pia mater, dura mater, falx, tentorium, cerebellum and brainstem) using only quadrilateral and hexahedral elements. Most of the existing FE models use tetrahedral elements (20-24). Theoretically speaking, tetrahedral elements have been known to be less accurate and less computationally efficient than hexahedral elements. Moreover, the human brain is an inhomogeneous and a highly complicated structure. To the best of our knowledge, no studies

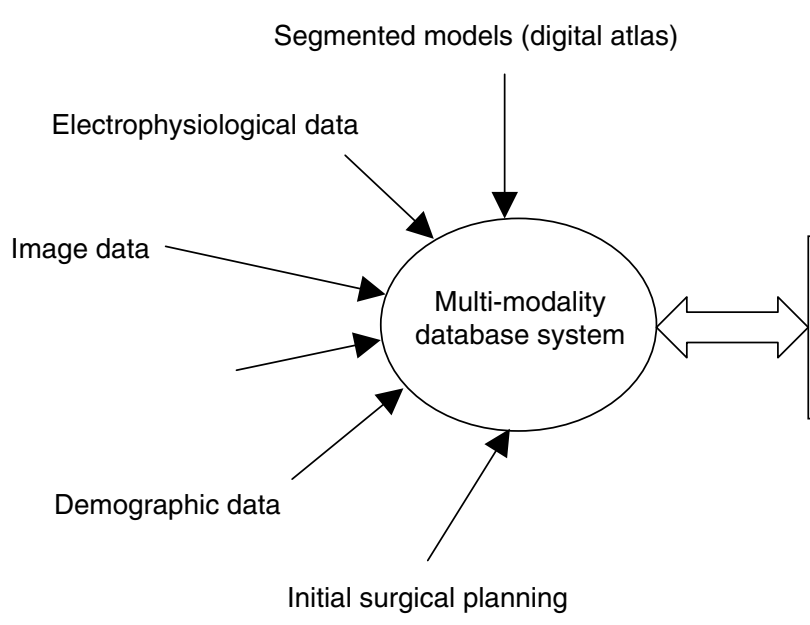

MULTI-MODALITY INPUT DATA

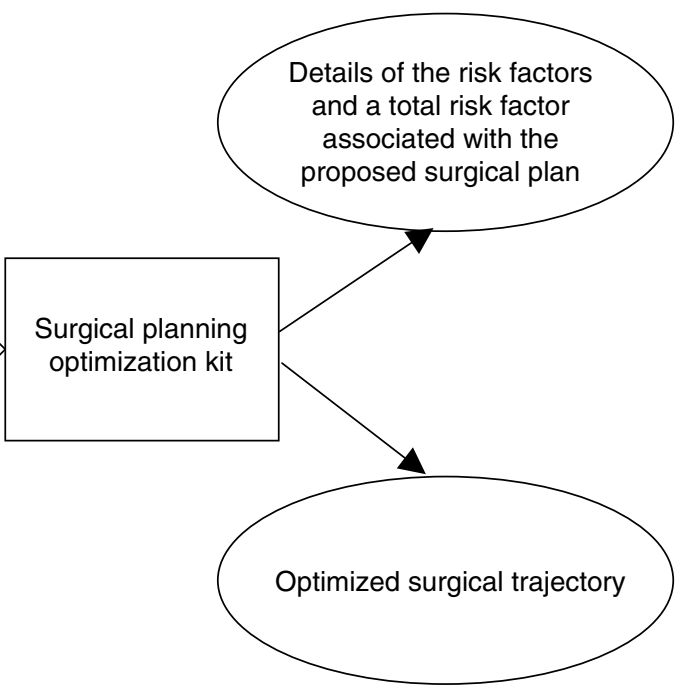

SYSTEM OUTPUT

Figure 6. CASMILDB overview 


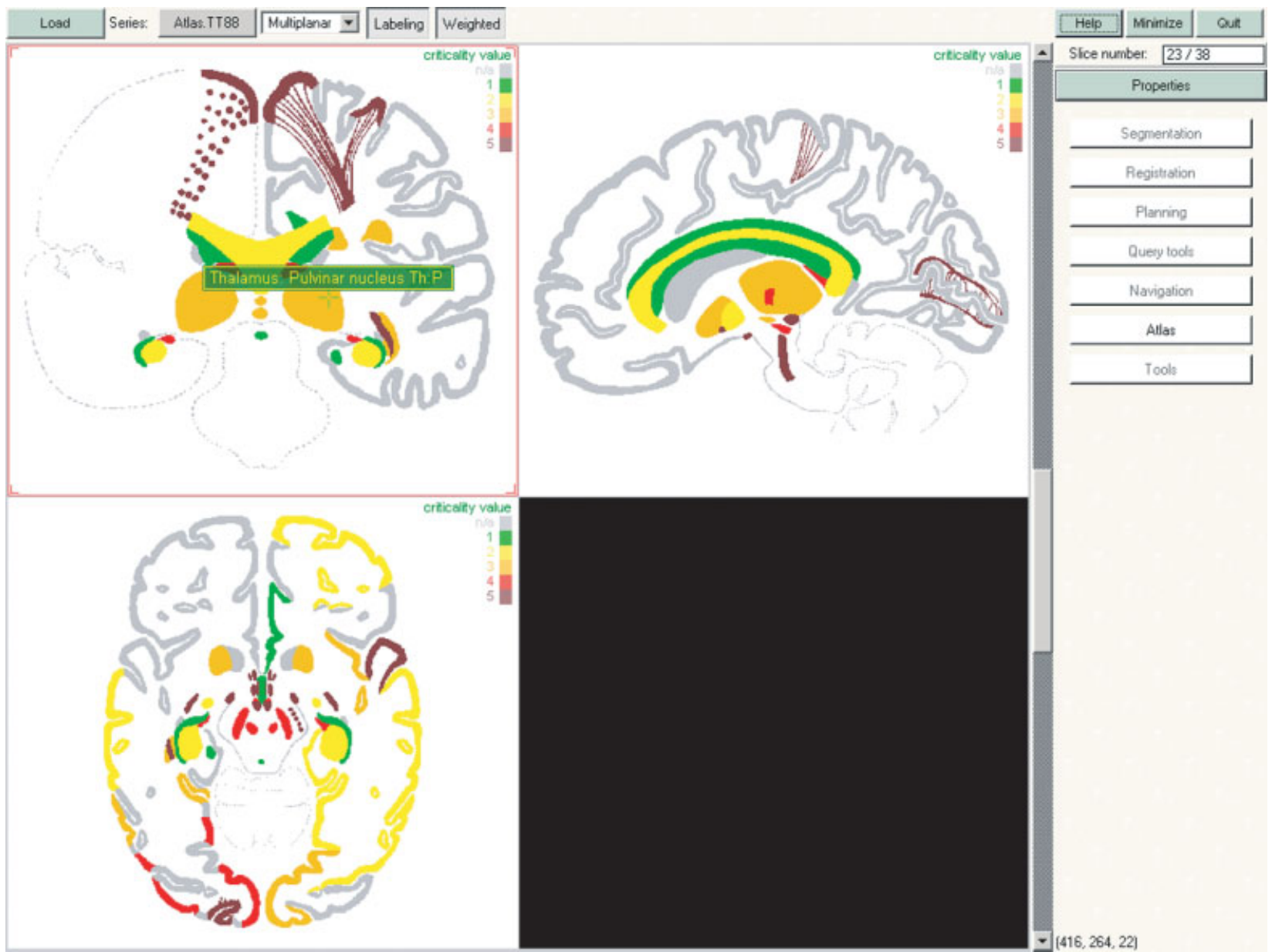

Figure 7. Weighted brain atlas
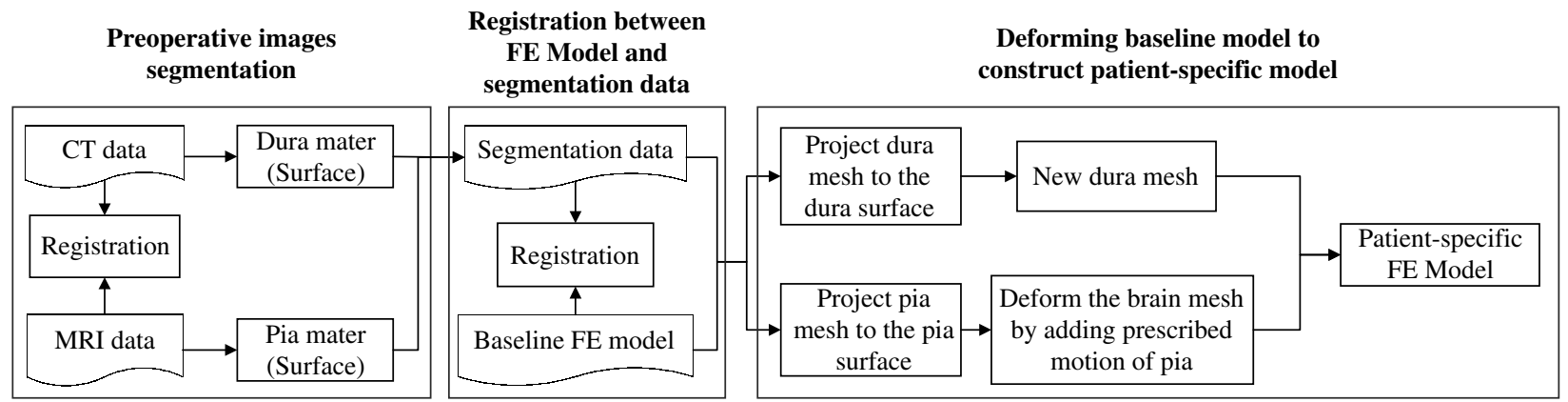

Figure 8. Patient-specific FE model generation algorithm

have been attempted to describe in detail the intracranial structures of the brain when calculating brain shift.

The following steps provide a brief description for the development of a patient-specific FE model and brain shift prediction:

1. Method to develop 3D patient-specific FE brain model (Figure 8).

a. A 3D FE brain model previously developed by Zhou et al. (25), as shown in Figure 9, is used as the baseline model.

b. Dura and pia mater are segmented for a specific patient. c. A landmark-based rigid registration is then used between the baseline model and segmented data, so as to compensate for global differences between the baseline model and the segmentation data.

d. To compensate for the local differences, the dura and pia mater of the baseline model are then projected onto the segmented dura and pia surface, respectively. The deformation vectors obtained through this process are used as boundary conditions (prescribed displacements) to deform the baseline model into the patient-specific model.

2. Different patient positions and different volumes of CSF drainage can be assumed, based on various surgery 


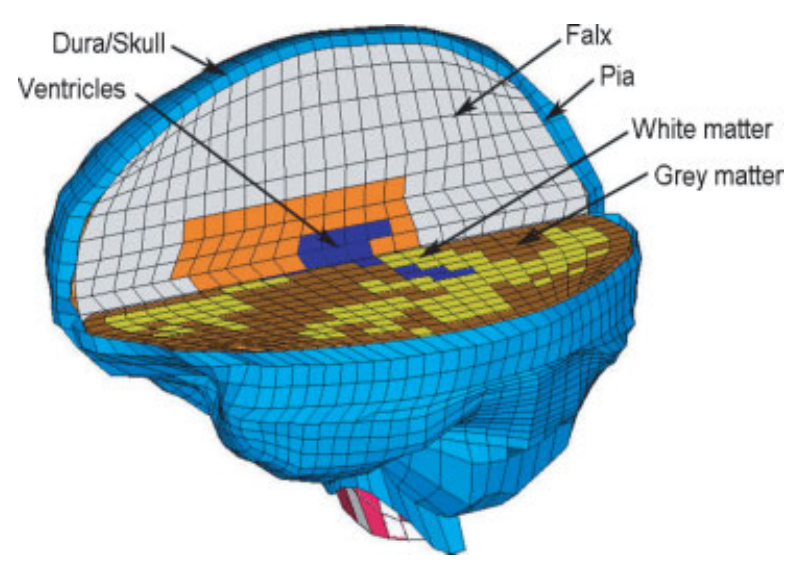

Figure 9. Oblique view of baseline FE human brain model

protocols, as shown in Figure 10. The arrows in the figure indicate the direction of gravity. Then the brain shift can be estimated by the patient-specific FE brain model.

3. After calculation, the preoperative MR images can be updated by the FE model results, so that surgeons can easily know the magnitude and direction of model-estimated brain shift. Figure 11 shows six landmark points selected to compare iMRI data and the numerical simulation. Landmark points one, three, four and five are located in the left ventricle, and landmark points two and six are located at sulci. The results are listed in Table 1 and are given in terms of the displacement measured by iMRI data and numerical simulations. In Figure 12 the light-coloured lines distinguish the boundaries of the brain before

Table 1. Comparison between the results of numerical simulations and IMRI data

\begin{tabular}{lcc}
\hline & \multicolumn{2}{c}{ Displacement $(\mathrm{mm})$} \\
\cline { 2 - 3 } Landmark \# & IMRI data & Numerical results \\
\hline 1 & 2.6 & 2.5 \\
2 & 6.2 & 7.3 \\
3 & 0.9 & 1.4 \\
4 & 2.0 & 4.1 \\
5 & 1.0 & 3.1 \\
6 & 6.2 & 6.5 \\
\hline
\end{tabular}

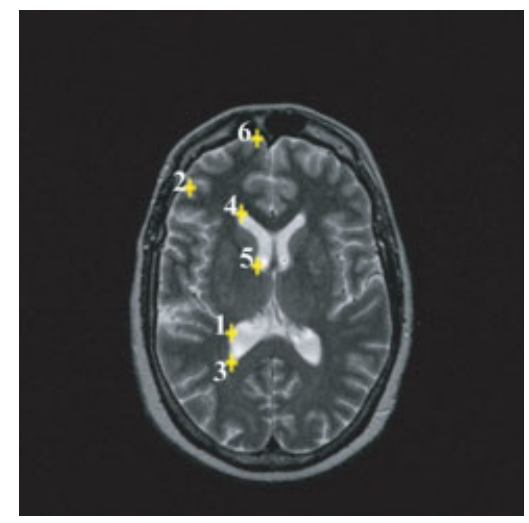

Figure 11. Six landmark points on the preoperative MR image

and after dura opening to highlight the magnitude of the brain shift. The maximum brain shift calculated in $3 \mathrm{D}$ space by the FE model was $7.3 \mathrm{~mm}$, while the maximum $2 \mathrm{D}$ brain shift predicted by the model in the transverse plane was $7.0 \mathrm{~mm}$. In the corresponding plane, the maximum displacement was $6.2 \mathrm{~mm}$ using iMRI.

We are also collecting brain tumour/lesion tissue specimens to measure their properties and integrate the resulting data into the FE model. Computationally intensive steps during brain shift prediction are being parallelized, thus providing near real-time results. This FE model will allow other institutions that do not have intraoperative MRI to benefit, and will also allow for better understanding of the mechanical properties of brain tissue.

\section{Intelligent planning}

CAS uses registered multi-modal images during a surgical procedure. A critical component of computer-assisted neurosurgery is preplanning, during which the surgeon plans a path of entry to a tumour that needs to be resected. The goal of this preplanning step is to find the shortest path that causes least damage. This becomes further challenging when the tumour is surrounded by vital structures or has an irregular shape. Slight damage

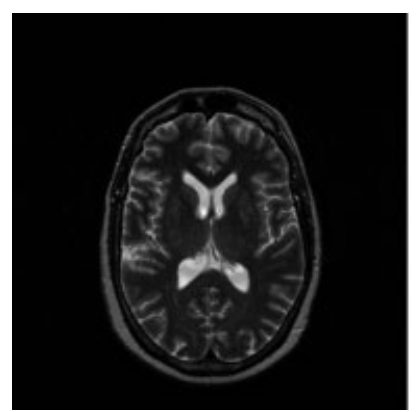

Before dura opening

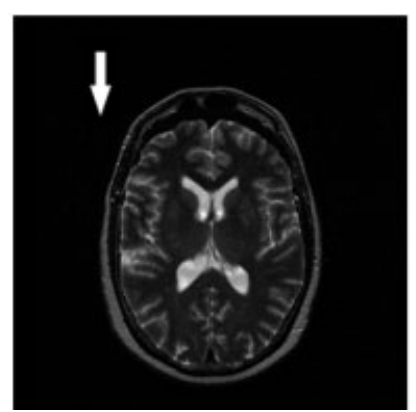

Supine position

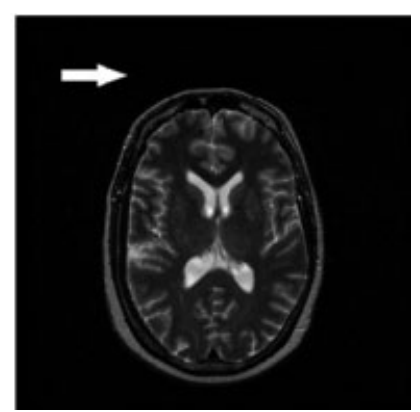

$90^{\circ}$ to the patient's right

After dura opening

Figure 10. Model updated images for different patient orientations 

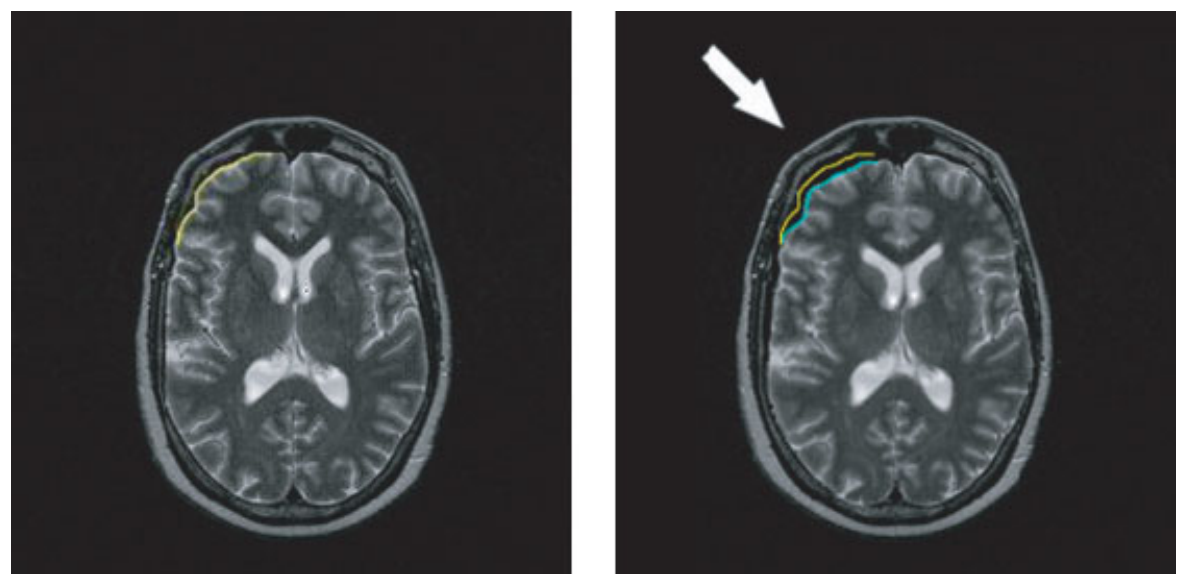

Figure 12. Preoperative MR image (left) and model updated MR image (right)

to critical structures can leave the patient highly impaired. CASMIL integrates an intelligent planning module, which provides a surgeon with a list of surgical corridors, based on the lesion location, shortest trajectory and critical brain structures. A key component of locating a surgical corridor is to find all brain structures that intersect with potential surgical trajectories that originate from a number of chosen points on the surface of the brain and end in the tumour. This module utilizes the surgeon's knowledge in terms of the weighted structures and acquired knowledge from previous cases in the database for decision-making and plan determination. Structures (137 in number) from the TT88 atlas have been segmented and given a priority value or weights by using surgeon knowledge and available functional data. The system performs a query based on potential surgical paths and weighted structures in the atlas, co-registered with patient images, and presents the surgeon with the shortest, least damaging corridor for tumour resection. This is beneficial not only during the preplanning phase but also during intraoperative navigation. If, during surgery, a surgeon is close to a critical region, such as the motor cortex, the system will display a message on the screen to avoid that region. The advanced intelligent planning module will integrate the patient-specific FE model. Thus, this module will provide the surgeon preoperatively with an updated surgical corridor based on predicted brain shift.

The query is performed using a novel indexing structure for spatial data in multi-dimensional space, called a target tree (26), designed to efficiently answer a new type of spatial query, called a radial query (26). A radial query seeks to find all objects in the spatial dataset that intersect with line segments emanating from a single, designated target point. Many existing and emerging biomedical applications use radial queries, including surgical planning in neurosurgery. Traditional spatial indexing structures such as the $\mathrm{R}^{*}$-tree and quadtree perform poorly on such radial queries. A target tree uses a regular hierarchical decomposition of space, using wedge shapes that emanate from the target point. A target tree is a variable-height tree that recursively decomposes the search space around a single target point. The index allows for insertion and deletion operations to be intermixed with searches. The tree itself may be stored on disk in a fashion similar to that of a quadtree or an octree. Target trees can be two-dimensional or three-dimensional (26). The target tree in 3D partitions space radially outward from the centre point in the same way as is done in the $2 \mathrm{D}$ case. In addition to the data stored in two dimensions, each tree node contains two $z$ dimension angles. The target tree can be stored and searched using a B+-tree by simply using the key for a target tree node, which is a (level, wedge-code) pair, as with the B+-tree index key. Our implementation uses this method.

A spatial object in a 3D space consists of a set of surface points which, when connected, form lines or polygonal surfaces that make up the boundaries of the region. We will consider inserting regions defined by some arbitrary number of such surface points in the search space into the target tree. The insertion algorithm progresses as follows. Starting at the root of the tree, the minimum bounding wedge (MBW) of the object to be inserted is checked to see which, if any, of the children of the root the object may lie within. The object is then inserted into any and all children whose space partition contains the MBW, either in whole or in part. This process recurses through the nodes in the tree until the object is inserted into a leaf or leaves that have space for it. If an object must be inserted into a leaf, and the leaf has reached a full capacity, the leaf is split, producing a new internal node in the tree and some number of new children, depending on the dimensionality. The new MBW, along with all the old MBWs that were contained in the old leaf, are inserted into any of the new children whose space partition intersects that of their respective MBWs.

To illustrate the insertion of a MBW into a target tree, consider inserting the object and MBW shown in Figure 13a into the tree represented in Figure 13b. The insertion algorithm begins by testing the four largest pieshaped wedges at level 2. Let R1, R2, R3, and R4 denote these wedges. Each of these wedges is checked to see whether it overlaps the MBW of the object being inserted. Only R1 (shown in 13a) overlaps with the MBW of the object, since the object lies entirely in the first quadrant. 


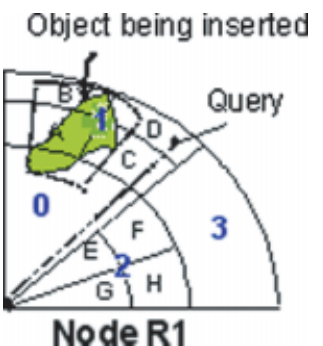

(a)

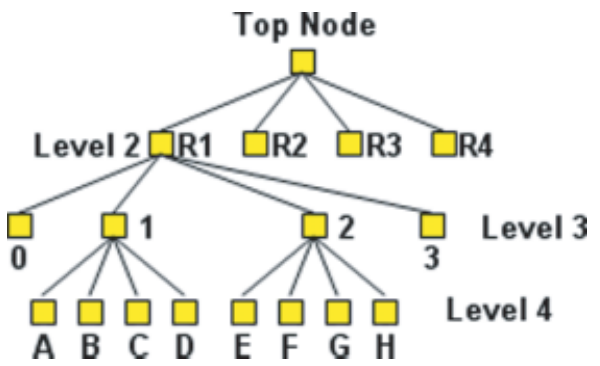

(b)

Figure 13. (a) An example of a MBW being inserted. (b) An example of a search query

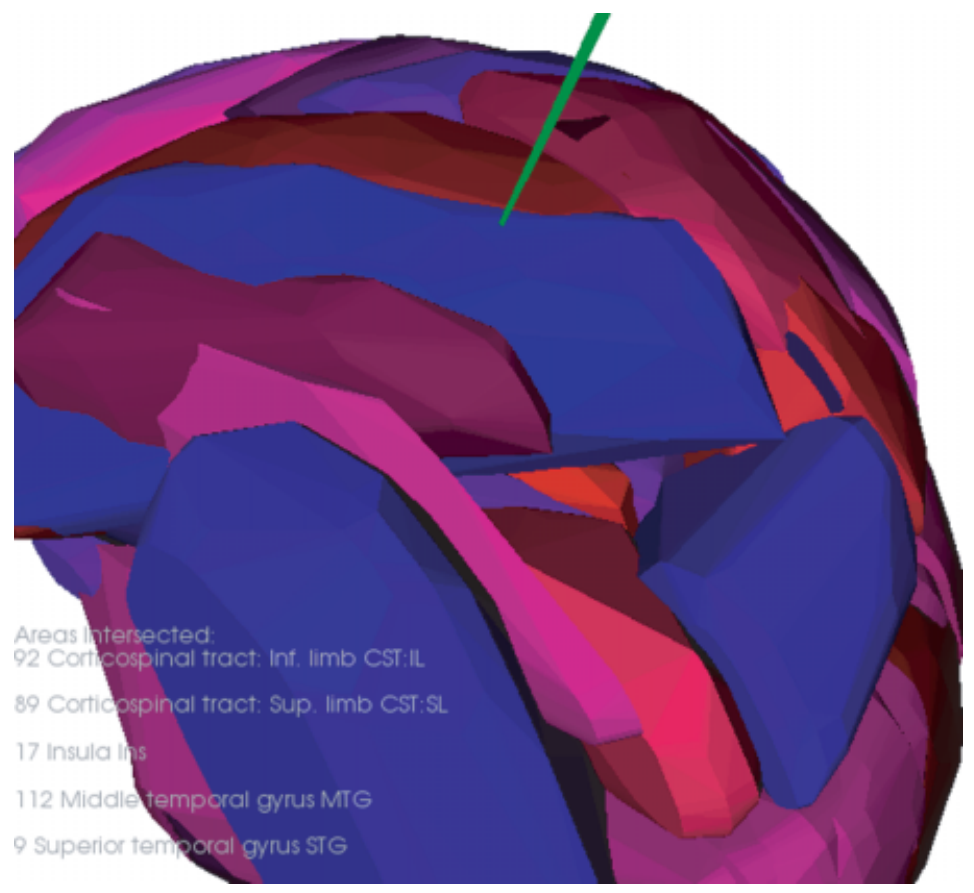

Figure 14. Snapshot of surgical corridor generated by intelligent planning module

Next, the four nodes at level 3 , i.e. the nodes labelled 0 , 1, 2 and 3 in Figure 13a, will be tested to see whether they overlap the MBW. Nodes 2 (which contains children E, F, G and $\mathrm{H}$ ) and 3 will be eliminated. Node 0 is a leaf, so it will include a reference to the object and its MBW. Node 1 is not a leaf, so the insertion will continue with its four children, A, B, C and D. Each overlaps the object's MBW and will have a reference placed in them. At the leaf level inserts, the insertion could have caused a further node split if the node fill threshold was exceeded.

We have conducted a detailed performance evaluation of the target tree compared with the $\mathrm{R}^{*}$-tree and quadtree indexing methods (27-29). Our experiments show that query evaluation with the target tree method outperforms these existing methods by at least a factor of five. We have also extended our approach to a cylindrical query path and have examined various techniques for query speed-up. Figure 14 shows different segmented features of the brain, in a red-blue scale, the trajectory that the database has computed, shown by the green arrow and a list of the structures that are intersected by the trajectory.

\section{Augmented reality}

CASMIL provides an improved and user-friendly surgeon-computer interface in the operating room by providing an augmented display of a virtual graphical image (such as a patient's segmented tumour) correctly registered with a view of the 3D real environment (such as the patient's head) (30). To date, surgeons have always been able to see the tumour and monitor its resection with $2 \mathrm{D}$ visualization. In order to continue along the pathway of smaller craniotomies and help surgeons to handle the huge amount of data he/she is dealing with, a 3D augmented reality, which preserves the geometrical 3D relationships between the objects of interest, has been developed (31). The patient image sequences are coregistered with the patient using the fiducial markers as the reference in the two coordinate spaces. This improved system generates a composite view for the surgeon that is a combination of the real scene and a virtual one that is generated by the computer. The computer-generated version is augmented with additional, surgically relevant information. Augmented reality is applied so that the surgical team 
can visualize the CT, MRI or other sensed data correctly registered on the patient in the operating room during a surgical procedure. Additional sensed data, such as functional MR (fMRI) images, could also be overlaid on this display. Providing this view to a surgeon in the operating room could enhance their performance. The ideal way to visualize and augment data would be to use intraoperative images to update any changed surgical information.

The above technology is developed to accurately show an 'X-ray' view of the objects of interest before opening the skull. The system consists of a video camera, an infrared (IR) camera and dynamic reference frames (DRFs). The DRFs are mounted on the camera and the skull phantom, which are tracked by the infrared camera, thus updating the position of the video camera in world coordinate space. The other alternative tracking technology used has been robotics and an articulated arm (30). Although the robotic digitizer provides better accuracy than infrared, its use in the operating room is restricted due to limited degrees of freedom. Independent of the tracking technology used, the AR system generates transformation matrices that can be solved to compute the position(s) and orientation(s) of the object(s) of interest in the camera coordinates. 3D models of objects of interest are acquired using the 3D segmentations techniques discussed above. These models are written in VTK polydata format. Headsup-display technology has also been integrated to the CASMIL, thus providing the surgeon with an augmented view of the real scene and graphical objects, via see-through or immersive glasses, rather than looking at the monitor continuously while performing a surgical procedure. We are undergoing human performance studies for qualitative and quantitative statistical analysis of the effects of wearing such headgear to perform various tasks during surgery.

\section{HPC for computationally intensive tasks}

Image-guided surgeries and therapies involve several computationally intensive steps, such as multi-modality image fusion, intelligent planning, 3D visualization, etc. In addition, the real-time analysis of complex data (image, sensed and electrophysiological data) and finite element modelling are extremely computationally intensive. Such computations cannot be executed on single-processor computers. Although voxel-based visualization serves a number of important uses in basic research, clinical diagnosis and treatment and surgery planning (32), it is limited by long rendering times for large image datasets and minimal interaction between the user and the rendered data. CASMIL utilizes high-performance computing for these computationally intensive tasks. It uses a message passing interface (MPI) for distribution of these tasks. MPI is the most widely used of the new standards in parallel computing (33). It is a library specification for message passing. Several nodes are used to distribute the workload across a grid network. Grid computing is an evolving area of distributed computing, where groups of computers share computing resources, such as memory, disk space and computing power, over a high-speed network. Grids are currently being developed for scientific and engineering research that requires large amounts of computation. Over the years, considerable progress has been made in grid architecture (34). Now, grid computing is emerging as a profitable area of computer science that can be used by corporations for computing other than research. The grid at Wayne State University allows access to 24 POWER-3 processors, 98 Pentium IV Xeon processors, eight Itanium- 2 processors with over 152 GB of RAM and over 4 TB of central disk space. It uses a high-speed, low-latency Myrinet switch fabric for communication. The coordinating node receives the entire image from CASMIL as well as instructions on what segmentation routines to perform on the images. The coordinator then sends individual slices to its nodes, which in turn run the appropriate routine on the image in parallel, and return the segmented images back to the coordinator. The coordinator then returns the entire segmented image back to the CASMIL. Segmentation of multiple 2D images can be done at one time, allowing for a rough estimate of the 3D segmentation, which can take up to 7 minutes. The resulting speed-ups have shown to be near-linear using image segmentation techniques such as Fuzzy Connectedness, Isolated Connectedness, Confidence Connected, Hybrid Fuzzy Voronoi, Geodesic, Neighbourhood Connected, Fast Marching and Connected Threshold (Figure 15). Using Fuzzy Connectedness to segment a stack of 50 images, the computation time was reduced from several minutes to only 16 seconds. CASMIL also takes advantage of multiple processors on each node using OpenMP, which on a dual-processor computer can double the speed. It also takes advantage of Intel's SSE and SSE2, which are Intel's single instruction multiple data (SIMD) extensions. These are essentially vector operations and ideally suited for several of the computations within CASMIL.

Currently we are implementing registration using MPI, OpenMP and SIMD instructions to drastically reduce the computation time. The results will be fast enough to allow for intraoperative, almost real-time, registration of preoperative image data to the low-quality intraoperative MRI.

\section{Web-enabled CASMIL}

Currently, the computer-assisted surgery (CAS) system is an isolated system in the operating room with obtrusive and cumbersome wired connections, which are not conducive to portable displays, or interactive surgical navigation and comparison to surgical plans. Surgery multi-modal data preparation, registration, segmentation, planning and related operations have to run and display at one physical site, which reduces the potential for collaborative interaction. There is a need for a more functional system that would enable surgeons at remote locations to access and plan the surgery, actively participate in remote surgeries, share patient information 


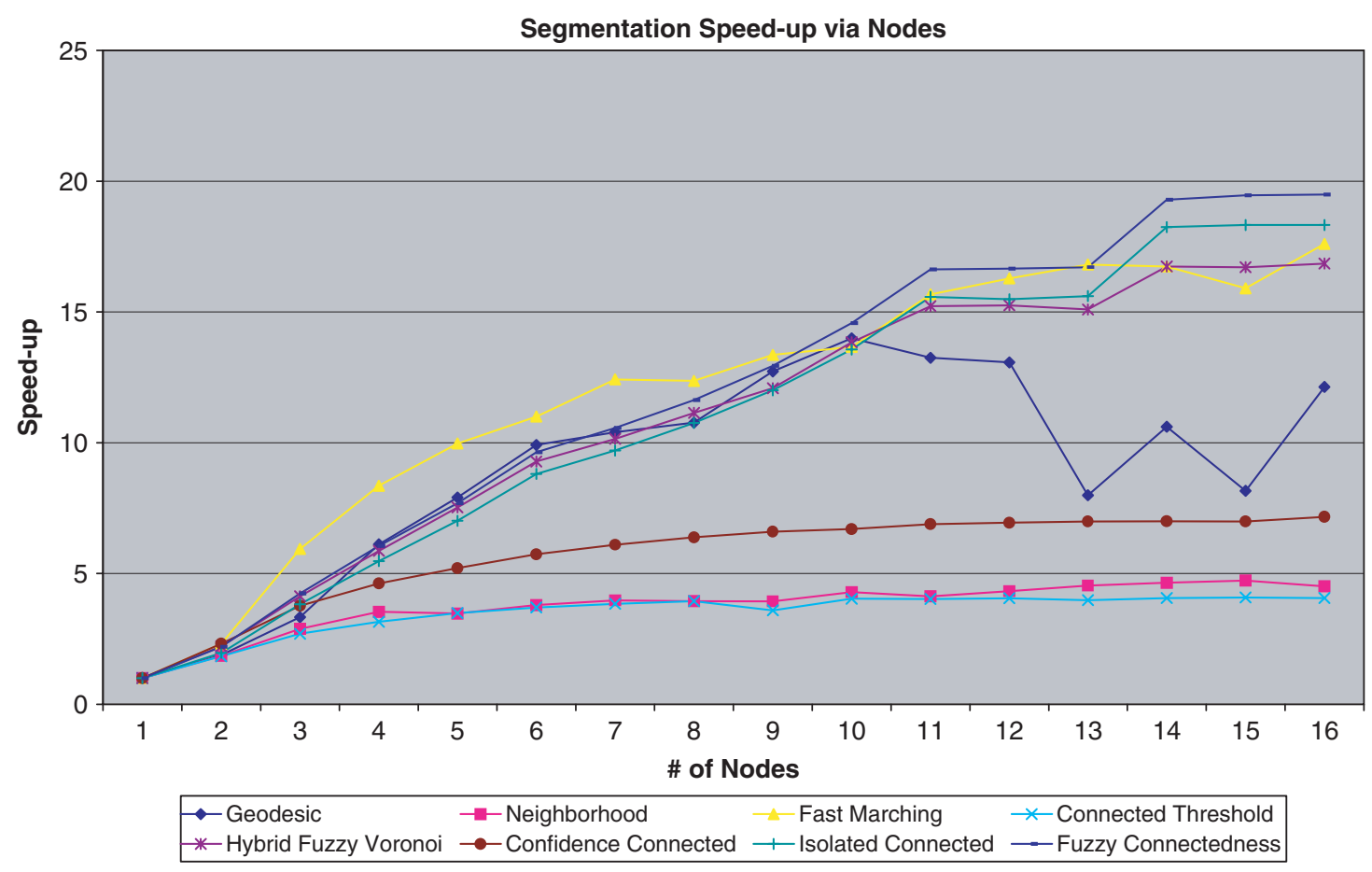

Figure 15. Segmentation speed-up for various segmentation methods

and exchange opinions in real time before, during and after surgery. The benefits of such an interaction may include providing access to highly specific and timecritical information for first responders in addition to more traditional in-theatre applications.

This project presents a collaborative environment for CAS, which will enable effective and efficient collaboration among multiple expert surgeons from different sites who have different roles in a surgery, using heterogeneous devices, such as desktop, laptop, PocketPC, etc.

With minimal changes to the standalone CASMIL, it has been made web-enabled and can be accessed anytime, anywhere by a surgeon using secured authentication mechanisms. Only some modules, such as co-registration, segmentation and planning, have been made webenabled. Other modules, such as augmented reality, cannot be made web-enabled because they require interaction with real-world (patient) coordinates. webenabled CASMIL provides flexibility to a surgeon to plan a surgery from his/her office or house. In addition, it is being optimized for easy remote access using PDA. With the popularity of wireless network and handheld devices, it has become necessary to bring CASMIL into these lightweight devices. Certain CASMIL modules, such as non-rigid registration, have to be moved to a backend server because of the constraints of computing and storage abilities of these devices. Only tasks that are not computationally intensive can be accomplished on handheld devices.

The client server architecture determines that communication across networks is a big issue for the web-enabled CASMIL. We systematically evaluated several of the most widely used network technologies for handheld devices, such as IEEE 802.11b, Bluetooth, dialup, Ethernet etc. Among them, some slow networks are becoming the obvious bottlenecks for data traffic on the network. In order to alleviate the effect of a slow network on the CASMIL, we have investigated several communication optimization techniques, such as Gzip, Vary-sized Blocking, Fix-sized Blocking, etc. (35), as well as the characteristics of the DICOM file, which is a very popular medical image processing format. A hybrid of the existing algorithms and the novel Bitmap algorithm (36), proposed by us specifically for DICOM images, can greatly reduce the traffic overhead to further eliminate the bottleneck if selected carefully according to different network scenarios and client configurations, as shown in Figure 16.

For a series of DICOM images which have to be transferred from server to client, Bitmap-Diff generates the smallest transfer bytes compared with other communication optimization mechanisms. This is significant for low-speed network users because the total delay is sensitive to the change of transfer bytes.

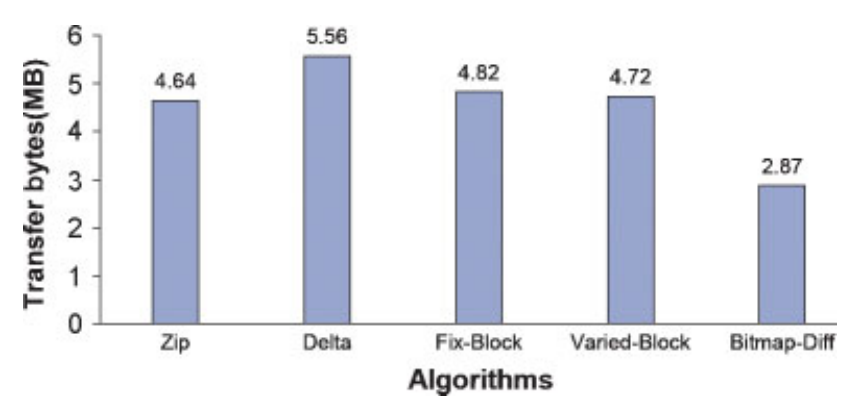

Figure 16. Transfer bytes of different communication algorithms 


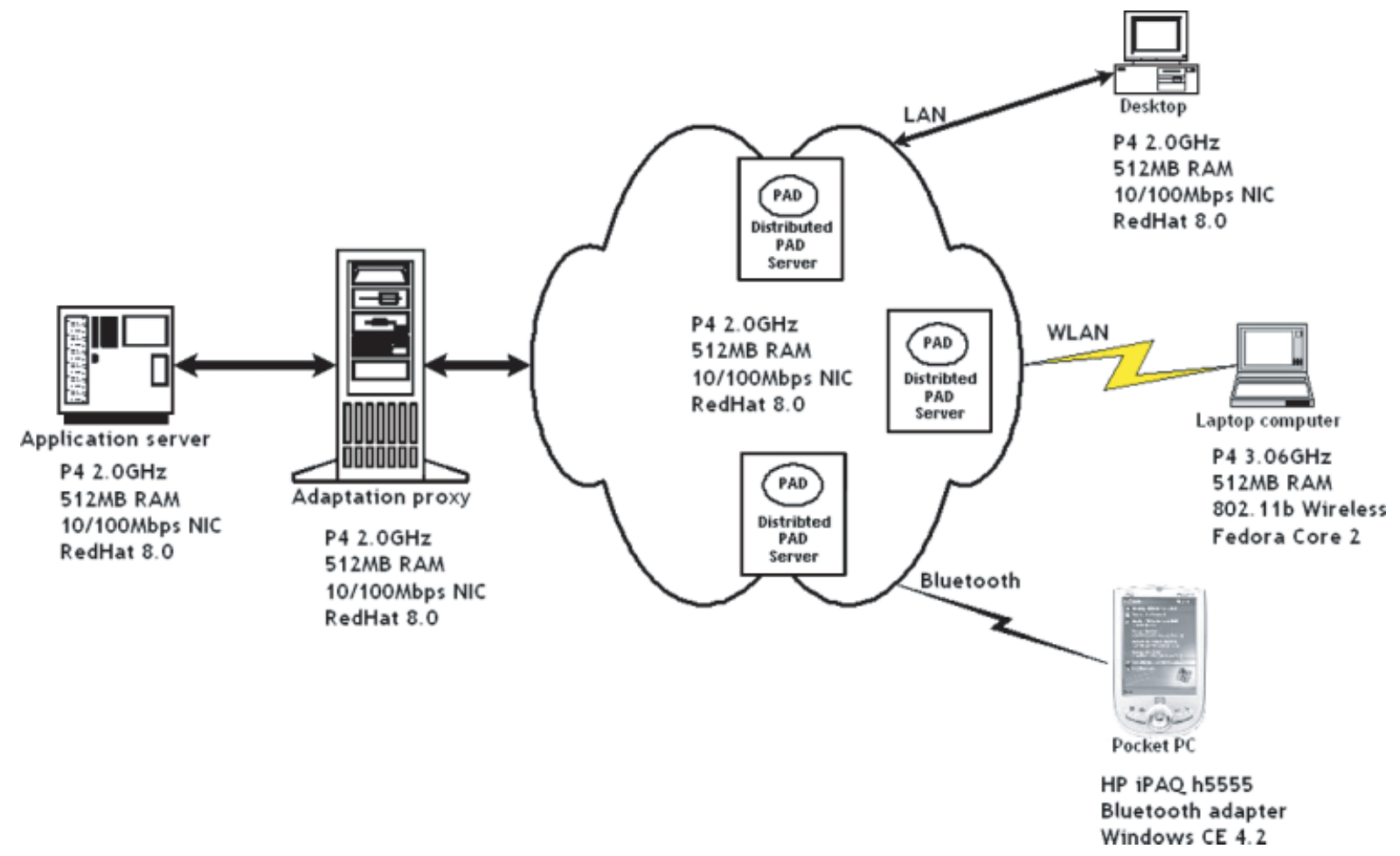

Figure 17. System architecture

In our Fractal framework (37), we have designed a comprehensive approach to the adaptation of communication optimization techniques for the CASMIL. The Fractal framework guides to dynamically choose the best communication optimization technique according to different client and network environments. The Fractal framework on the communication optimization for web-enabled CASMIL will dramatically reduce the communication time across different networks. In Fractal, if we consider each communication optimization technique as a protocol adaptor (PAD) for a specific application, one of the techniques, a PAD, will be chosen in different scenarios. Four PADs used in CASMIL are Durect, Gzip, Var-sized and Bitmap.

As shown in the system architecture (Figure 17), different clients, such as desktop, laptop and PDA, negotiate with the adaptation proxy to find the appropriate PAD. The adaptation proxy then informs the client about the metadata of this PAD, based on which the client is able to download it from the content delivery network (CDN). Then, under the adaptation of the PAD, client and server efficiently set up the application communication session. One of the experiment results is shown in Figure 18.

In Figure 18, the $x$ axis represents the different client configurations and the $y$ axis is the total time used by the different communication optimization algorithms selected by the three kinds of adaptation mechanism. For instance, for PDA in Bluetooth, no protocol adaptation mechanism chooses direct sending as the optimization algorithm, as shown by the green bar. Its total time is high. The fixed protocol adaptation mechanism always

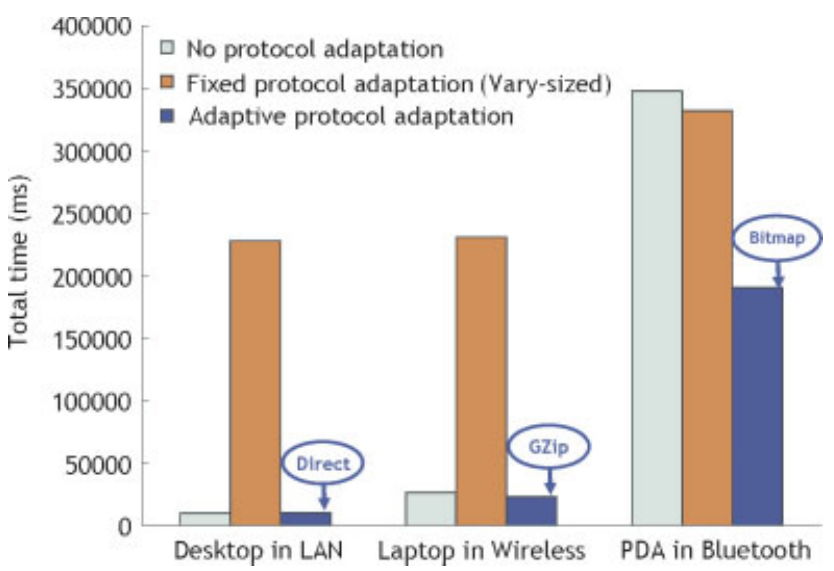

Figure 18. Bytes transferred by different adaptation mechanism for different client configurations

chooses the Varied-Block optimization algorithm, which also has a high total time. However, the adaptive protocol adaptation mechanism in Fractal can choose Bitmap as the optimization algorithm, which tenders the smallest total time delay that the user could experience.

The experiment proves that the Fractal framework is especially suitable for applications that require dynamic application protocol adaptation flexibility, such as webenabled CASMIL.

\section{Comparison with Other Toolkits}

Most of the existing image-guided systems provide multiplanar and 3D views, basic registration and segmentation 
Table 2. Comparison of the existing image-guided systems for neurosurgery

\begin{tabular}{|c|c|c|c|c|c|c|}
\hline & Non-rigid registration & $\begin{array}{c}3 \mathrm{D} \\
\text { segmentation }\end{array}$ & $\begin{array}{l}\text { Brain shift } \\
\text { predictor }\end{array}$ & $\begin{array}{l}\text { Intelligent } \\
\text { planning }\end{array}$ & $\begin{array}{l}\text { Query } \\
\text { tools }\end{array}$ & Augmented reality \\
\hline CASMIL & Yes & Yes & Yes & Yes & Yes & Yes \\
\hline IGSTK & $\begin{array}{l}\text { Organ model to image } \\
\text { registration, and } \\
\text { fluoroscopy (video input) } \\
\text { to CT registration }\end{array}$ & Yes & No & No & No & $\begin{array}{l}\text { Tracker: } \\
\text { electromagnetic, } \\
\text { optical }\end{array}$ \\
\hline Analyse Direct & Yes & Yes & No & No & No & No \\
\hline Stryker & No & Near-3D* & No & No & No & Yes \\
\hline Medtronics & No & Yes (tumour) & Yes (tumour) & No & No & No \\
\hline Brain Lab & Z-touch laser registration & Yes & No & No & No & Yes \\
\hline Atamai/Robarts Imaging & Yes & No & No & No & No & $\begin{array}{l}\text { Optical and magnetic } \\
\text { tracking }\end{array}$ \\
\hline Surgical Planning Laboratory** & Yes & No & Yes & No & No & Yes \\
\hline The Computer-Aided Surgery Laboratory*** & Yes & No & No & No & No & Yes \\
\hline
\end{tabular}

*Using multi-slice 2D segmentation to simulate 3D segmentation.

***Bigham and Women's Hospital.

*** Centre of Biomedical Engineering and Physics at the Medical University of Vienna.

techniques, planning of surgical corridors and navigation (38-48). In these systems, preoperative planning is performed by neurosurgeons by defining the entry and the target points, thus forming a trajectory for the surgical instrument.

As shown in Table 2, CASMIL has the most comprehensive features. Some toolkits have non-rigid registration and 3D segmentation, such as IGSTK, Analyse Direct and Brain Lab. However, they lack the brain shift predictor, intelligent planning and query tools modules. Surgical Planning Laboratory and Medtronics imageguided systems have brain shift predictors but they do not include intelligent planning and query tools modules.

CASMIL, in addition to these basic functionalities, includes an intelligent planning module, which provides the surgeon with a list of optimum surgical corridors, based on the lesion location, shortest trajectory and extensive surgeons' knowledge in terms of critical brain structures and predicted brain shift, using the patient-specific finite element model (see Table 2). Other vital modules of this system include 3D segmentation, query tools and augmented reality. High-performance computing (HPC) is used to speed up computationally intensive tasks involved in CAS, thus providing near realtime results while performing surgery. This system has also been made securely web-enabled for surgeons to access it from any other location (e.g. their homes or offices). In addition, the system is being optimized for remote PDA access. Another unique feature of this system is the integration of various digital brain atlases.

\section{Conclusion}

We have described the design and features of a new image-guided neurosurgery tool, CASMIL. Clearly, it has many features that are not available in other academic and commercial tools currently being used for neurosurgeries. CASMIL is still under continual validation and development. Feedback from surgeons and clinical engineers using the system is being used to improve the system. We have tested current modules of CASMIL using 10 cases from the Detroit Medical Centre.

\section{References}

1. Gong J, Zamorano L, Li Q, Diaz F. Intraoperative neuronavigation and surgeon interface. Med Imaging 1999.

2. Zamorano L, Nolte LP, Kadi AM, Jiang Z. An interactive intraoperative localization using an infrared-based system. Stereotact Funct Neurosurg 1994; 63: 84-88.

3. ITK Software Guide: www.itk.org/ItkSoftwareGuide.pdf

4. Thirion JP. Fast non-rigid matching of 3D medical images. Technical Report, Research Report RR-2547, Epidural Project, The French national Institute for Research in Computer Science and Control (INRIA) Sophia, May 1995.

5. Thirion JP. Image matching as a diffusion process: an analogy with Maxwell's demons. Med Image Anal 1998; 2(3): 243-260.

6. Bookstein FL. Morphometric Tools for Landmark Data: Geometry and Biology. Cambridge University Press: New York, 1992.

7. Miller M, Trouve A, Younes L. On the metrics and EulerLagrange equations of computational anatomy. Annu Rev Biomed Eng 2002; 4: 375-405.

8. Bajcsy R, Broit R. Matching of deformed images. In $6^{\text {th }}$ International Conference on Pattern Recognition. Munich, Germany 1982; 351-353.

9. Nowinski WL, Bryan RN, Raghavan R. The Electronic Clinical Brain Atlas. Multiplanar Navigation of the Human Brain. Thieme: New York, Stuttgart, 1997.

10. Schaltenbrand G, Wahren W. Atlas for Stereotaxy of the Human Brain. Thieme: Stuttgart, 1977.

11. Talairach J, Tournoux P. Referentially Oriented Cerebral MRI Anatomy. Atlas of Stereotaxic Anatomical Correlations for Grey and White Matter. Thieme: Stuttgart, New York, 1993.

12. Talairach J, Tournoux P. Co-Planar Stereotactic Atlas of the Human Brain. Thieme: Stuttgart, New York, 1988.

13. Nowinski WL. Analysis of medical images by means of brain atlases. Comput Graphics Vision 1999; 8(3): 449-468.

14. Nowinski WL. Modified Talairach landmarks. Acta Neurochir 2001; 143: 1045-1057.

15. Dingguo C. Technical reports on image processing, year II, second and third quarters, 2005; Computer Aided Surgery Laboratory, Wayne State University: http://www.caslmlsc.med.wayne.edu/

16. Geiger B. Three-dimensional modelling of human organs and its application to diagnosis and surgical planning. Technical Report RR No. 2105. Institut National de Recherche en Informatique et en Automatique (INRIA): Le Chesnay Cedex, France 1993. 
17. Melax S. A simple, fast and effective polygon reduction algorithm. Game Developers Mag 1998; 11: 44-49.

18. Lee JB, Hu J, Zhang L, et al. A parametric study on gravityinduced brain shift using a three-dimensional FE model of the human brain. Proceedings of ASME International Mechanical Engineering Congress and Exposition (IMECE 2004-61006), 13-20 November 2004, Anaheim, CA, 2004; 13-20.

19. Lee JB, Hu J, Zhang L, et al. Investigation of gravity-induced brain shift based on a three-dimensional finite element model of the human brain. In Proceedings of the Congress of Neurological Surgeons Annual Meeting, 16-21 October 2004,San Francisco, CA, 2004.

20. Ferrant M, Nabavi A, Macq B, et al. Serial registration of intraoperative MR images of the brain. Med Image Anal 2002; 6: 337-359.

21. Ferrant M, Warfield SK, Guttmann CRG, et al. 3D image matching using a finite element based elastic deformation model Proc Med Image Comput Computer Assist Intervent. Springer LNCS: New York, USA, 1999; 202-209.

22. Miga MI, Roberts DW, Kennedy FE, et al. Modelling of retraction and resection for intraoperative updating of images. Neurosurgery 2001; 49(1): 32-37.

23. Miga MI, Paulsen KD, Lemery JM, et al. Model-updated image guidance: initial clinical experiences with gravity-induced brain deformation. IEEE Trans Med Imaging 1999; 18(10): 866-874.

24. Smith-Castellano A, Hartkens T, et al. Constructing patientspecific models for correcting intraoperative brain deformation. Proc Med Image Comput Computer Assist Intervent. Springer LNCS: New York, USA, 2001; 1091-1098.

25. Zhou C, Khalil TB, King AI. A new model comparing impact responses of the homogeneous and inhomogeneous human brain. Proceedings of the 39th Stapp Car Crash Conference, Society of Automotive Engineering (SAE) Paper No. 952714. SAE: Warrendale, PA, 1995.

26. Morse M, Patel JM, Grosky W. Efficient evaluation of radial queries using the target tree. In International Workshop on Biomedical Data Engineering (BMDE), 2005; p 1168.

27. Beckmann N, Kriegel H-P, Schneider R, Seeger B. The R*-tree: an efficient and robust access method for points and rectangles. In Special Interest Group on Management of Data (SIGMOD), 1990; 322-331.

28. Guttman A. R-trees: a dynamic index structure for spatial searching. In Special Interest Group on Management of Data (SIGMOD), 1984; 47-57.

29. Samet $H$. The quadtree and related hierarchical data structures. Comput Surveys 1984; 16(2): 187-260.

30. Zamorano L, Pandya A, Siadat M, et al. Tracking methods for medical augmented reality. Med Image Comput Computer Assist Intervent 2001; 1404-1405.

31. Alam M, Daryan L. An augmented reality system to enhance intraoperative visualization for computer-assisted neurosurgery. Abstract accepted for poster, Medical Meets Virtual Reality 13 (MMVR-13), CA.

32. Robb RA. Three-dimensional Biomedical Imaging - Principles and Practice. VCH: New York, 1995.
33. Warfield SK, Jolesz FA, Kikinis R. Real-time image segmentation for image-guided surgery. In Super Computing 1998. IEEE Computer Society: 1998

34. Ian TF. The anatomy of the grid: enabling scalable virtual organizations. Proceedings of the $7^{\text {th }}$ International Euro-Par Conference on Parallel Processing, Manchester, 2001. SpringerVerlag: New York, USA, 2001; 1-4.

35. Lufei $\mathrm{H}$, Shi W, Zamorano L. On the effects of bandwidth reduction techniques in distributed applications. In International Conference on Embedded and Ubiquitous Computing (EUC-04), August, Aizu, Japan.

36. Lufei H, Shi W, Zamorano L. Communication optimization for image transmission in computer-assisted surgery. Accepted by the 2004 Congress of Neurological Surgeons Annual Meeting (abstract), October 2004, San Francisco, CA.

37. Lufei H, Shi W. Fractal: a mobile code based framework for dynamic application protocol adaptation in pervasive computing. In $19^{\text {th }}$ IEEE International Parallel and Distributed Processing Symposium, Denver, CO, April 2005 (The Best Paper Award).

38. Cleary K, Ibanez L, Ranjana S, Blakec B. IGSTK: a software toolkit for image-guided surgery applications. Comput Aided Radiol Surg 2004; 1268: 473-479.

39. Dey D, Gobbi DG, Slomka PJ, et al. Automatic fusion of freehand endoscopic brain images to three-dimensional surfaces: creating stereoscopic panoramas. IEEE Trans Med Imaging 2002; 21(1): 23-30.

40. Foley KT. StealthStation: latest technological advances. New Frontiers in Spine Surgery Meeting, Strasbourg, France, October 1997.

41. Grimson WEL, Ettinger GJ, Kapur T, et al. Utilizing segmented MRI data in image-guided surgery. In International Journal of Pattern Recognition and Artificial Intelligence (IJPRAI), 1996.

42. Grimson WEL, Perez TL, Wells WM III, et al. An automatic registration method for frameless stereotaxy, image guided surgery, and enhanced reality visualization. Trans Med Imaging 1996; 129-140.

43. Kirk W, Finnis YP, Starreveld AG, et al. A 3-dimensional database of deep brain functional anatomy, and its application to imageguided neurosurgery. MICCAI-2000: $1-8$.

44. Peters TM. Image-guided surgery: from X-rays to virtual reality. Comput Methods Biomech Biomed Eng 2000; 4(1): 27-57.

45. Wagner A, Schicho K, Birkfellner W, et al. Quantitative analysis of factors affecting intraoperative precision and stability of optoelectronic and electromagnetic tracking systems. Med Phys 2002; 29(5): 905-912.

46. Analyse Direct. Comprehensive Visualization for Biomedical Imaging: http://www.analysedirect.com/Analyse/

47. Brain Lab Vector Vision and the Navigus: http://www.brainlab. com/scripts/website_english.asp

48. Stryker Leibinger: http://www.stryker.com/navigation/neuro/ index.htm 